\title{
Aircraft Conceptual Design and Risk Analysis Using Physics-Based Noise Prediction
}

\author{
Erik D. Olson* \\ NASA Langley Research Center, Hampton, VA, 23681 \\ Dimitri N. Mavris ${ }^{\dagger}$ \\ Georgia Institute of Technology, Atlanta, GA, 30332
}

\begin{abstract}
An approach was developed which allows for design studies of commercial aircraft using physics-based noise analysis methods while retaining the ability to perform the rapid tradeoff and risk analysis studies needed at the conceptual design stage. A prototype integrated analysis process was created for computing the total aircraft EPNL at the Federal Aviation Regulations Part 36 certification measurement locations using physics-based methods for fan rotor-stator interaction tones and jet mixing noise. The methodology was then used in combination with design of experiments to create response surface equations (RSEs) for the engine and aircraft performance metrics, geometric constraints and takeoff and landing noise levels. In addition, Monte Carlo analysis was used to assess the expected variability of the metrics under the influence of uncertainty, and to determine how the variability is affected by the choice of engine cycle. Finally, the RSEs were used to conduct a series of proof-of-concept conceptual-level design studies demonstrating the utility of the approach. The study found that a key advantage to using physics-based analysis during conceptual design lies in the ability to assess the benefits of new technologies as a function of the design to which they are applied. The greatest difficulty in implementing physics-based analysis proved to be the generation of design geometry at a sufficient level of detail for high-fidelity analysis.
\end{abstract}

\section{Nomenclature}

Response surface equation coefficient

Drag coefficient

Drag coefficient at zero lift

Lift coefficient

Lift coefficient at zero angle of attack

Lift-curve slope

D criterion

Fan diameter

Blade camber surface function

Objective function

$F_{\text {shock }} \quad$ Shock pressure loss factor

$k \quad$ Turbulence intensity

$K_{1} \quad$ Linear coefficient of drag polar equation

$K_{2} \quad$ Quadratic coefficient of drag polar equation

$\dot{m} \quad$ Mass flow rate

$n \quad$ Number of design variables

$n \quad$ Number of streamlines

$\Delta p \quad$ Fan blade static pressure differential

\footnotetext{
*Aerospace Engineer, Aeronautics Systems Analysis Branch, AIAA Senior Member.

${ }^{\dagger}$ Boeing Professor, School of Aerospace Engineering, AIAA Associate Fellow.
} 


$\begin{array}{ll}p_{r, d e s} & \text { Design fan rotor total pressure rise } \\ p_{r, i} & \text { Total pressure on the } i^{\text {th }} \text { streamline } \\ r & \text { Radius } \\ R & \text { Response value } \\ V_{r} & \text { Radial velocity component } \\ V_{z} & \text { Axial velocity component } \\ V_{\theta} & \text { Circumferential velocity component } \\ V_{\theta}^{*} & \text { Prescribed circumferential velocity distribution } \\ x & \text { Generic design variable } \\ \bar{x} & \text { Normalized value of design variable } \\ \mathbf{X} & \text { Design matrix } \\ z & \text { Axial coordinate } \\ z_{s h o c k} & \text { Axial shock location relative to blade chord } \\ \alpha & \text { Angle of attack } \\ \alpha_{c} & \text { Empirical constant } \\ \beta_{c} & \text { Empirical constant } \\ \Omega & \text { Fan rotational speed }\end{array}$

\section{Introduction}

In the near future, worldwide demand for air travel is expected to grow by an average of about 4 percent per year, so that by 2040 the demand is expected to be about four times as great as today. ${ }^{1}$ Although today's aircraft are $20 \mathrm{~dB}$ quieter than the first jet-powered airplanes, earlier dramatic improvements in noise levels have gradually given way to smaller improvements, so that the trend of lower noise with time in recent years has approached a slope of zero. If noise reduction does not continue, noise restrictions at the international, national and local levels could severely constrain the capacity of the global aviation system to meet the growing demand. ${ }^{2,3} \mathrm{~A}$ recent survey found that the majority of European airports are already subject to direct noise-related constraints on capacity, and the percentage is expected to increase significantly in the near future. ${ }^{4}$

Prior experience has shown that the noise level of any single source can be reduced by a few decibels through conventional design alternatives, but achieving additional reductions requires a significantly larger level of effort in terms of research and cost and an increasingly interdisciplinary effort. ${ }^{5}$ Thus, it will become necessary to focus not just on the reduction of individual noise sources, but on a concurrent approach which examines the interaction of multiple engineering disciplines at earlier stages in the design process.

\section{A. First-Principles Noise Analysis}

Adopting a multidisciplinary analysis environment and improving the fidelity of analysis in the early stages of the design process result in a more rapid increase in design knowledge than in the traditional design process. Additionally, by limiting the number of choices made about the design until more knowledge can be obtained, the design freedom can be retained for a greater portion of the process so that the greatest drop-off in freedom occurs at the later design stages.

During a traditional conceptual design study, however, the limited information about the engine geometry and flow fields usually necessitates the use of empirical methods to predict the acoustic characteristics of the design. Unfortunately, empirical noise prediction methods can be extremely limited because the database which makes up the empirical curve fits may be outdated in terms of the technology level and design methods, so any empirical database can quickly become unsuitable for examining revolutionary design concepts and technologies.

On the other hand, analysis methods based on physical laws, i.e. first principles, are always applicable to any design problem - at least in theory - because the laws of physics always apply. Additionally, firstprinciples analysis (FPA) tends to give better trends outside the empirical database because it is not subject to extrapolation errors like empirical analysis, and can give a better assessment of the effects of variables which are not included in the empirical equations. Another very important advantage of FPA is the ability to make a more direct and thorough analysis of new technologies by direct simulation rather than correction 
factors.

The drawbacks to using FPA are that the analysis is by necessity much more complex than can typically be afforded early in the design process, requires a much more thorough definition of the engine geometry and internal flow field than is usually available for a conceptual design, and requires a much greater execution time, making it impossible to perform the large numbers of analyses required in numerical optimization.

\section{B. Noise Guarantees}

An airframe manufacturer is typically required to guarantee to a customer that its aircraft will not exceed maximum allowable community, interior, and ramp noise levels. These guarantees are often a very important factor in competition among airplane and engine manufacturers. ${ }^{6}$ Unfortunately, it is impossible to predict perfectly the measured noise levels of the aircraft due to inherent uncertainties that are always present. To account for the uncertainty in the final noise levels, the aircraft must be designed so that the nominal noise prediction is sufficiently below the guarantee levels to reduce the design risk to an acceptable level. Increased fidelity in noise analysis early in the design process could reduce the overall uncertainty, allowing for smaller initial margins for the same design risk.

\section{Goal}

The fundamental goal for this research was to develop a methodology for the conceptual design and risk analysis of quiet commercial aircraft using noise analyses which are based on first principles. Techniques were sought out which make it practical to incorporate higher-fidelity noise analysis into early design stages, thus improving the knowledge which can be gained about the design, while still retaining the flexibility to examine many different combinations of design variables so as not to limit the design freedom. In addition, methods were used for estimating the variance in the overall noise levels due to the presence of underlying uncertainty, and for examining the effect of design variables on the overall noise risk.

\section{Background}

Certification noise levels are measured and regulated under the requirements of the International Civil Aviation Organization (ICAO) Annex 16, Chapter 4 noise regulations, and in the United States under the Federal Aviation Regulations (FAR) Part 36, Stage $4 .^{7}$ An aircraft is flown through standardized takeoff and approach procedures, and the 1/3-octave band frequency spectrum time histories are measured at three locations: sideline and takeoff locations during the takeoff maneuver, and an approach location during the approach maneuver (Figure 1(a)). The unit of measurement for certification noise is the effective perceived noise level (EPNL), and the maximum noise levels for the three measurement locations are specified as a function of maximum takeoff weight and the number of engines. The maximum EPNLs for the three locations are shown in Figure 1(b) for Stage 3 certification. As of January 1, 2006, all new commercial aircraft are required to meet ICAO Chapter 4 noise limits, ${ }^{8}$ which require that (a) Stage 3 limits are not exceeded at any measurement location, (b) the sum of the differences between the Stage 3 limit and the measured noise level at the three locations is at least $10 \mathrm{EPNdB}$, and (c) the sum of the differences is at least $2 \mathrm{EPNdB}$ for any pair of locations.

\section{Analysis Process}

A prototype integrated analysis process was created for computing the relationship between the engine and aircraft design and uncertain variables to the total aircraft EPNL at the FAR 36 certification measurement locations; a description of the initial development of the analysis process was published previously. ${ }^{9}$ See Figure 2(a) for a flow chart of the analysis process, including the analysis steps and the data requirements at each step. The Source Noise Analysis block in the flow chart is shown in greater detail in Figure 2(b). Each of the components of the analysis process are described in the following sections.

\section{A. Engine Performance}

The engine cycle analysis program ENGGEN ${ }^{10,11}$ is used to compute the design and off-design steady-state performance and thermodynamic properties of the engine. ENGGEN is based on the QNEP program, ${ }^{12}$ 


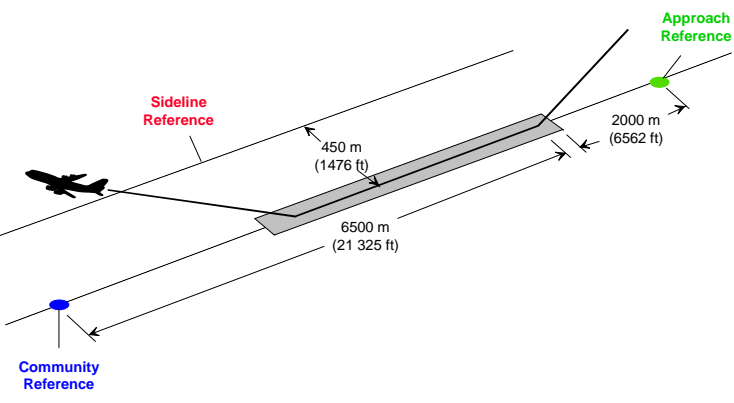

(a) observer locations

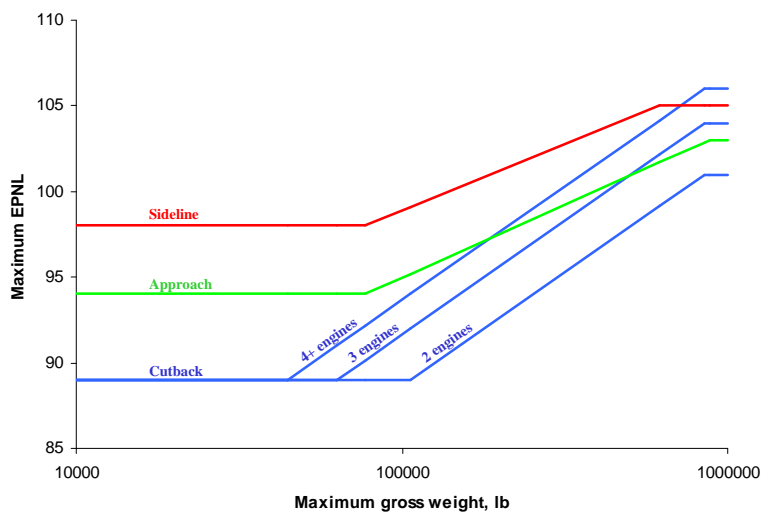

(b) Stage 3 noise limits

Figure 1. Observer locations and Stage 3 noise limits for FAR 36 certification. The sideline observer is located along the sideline at the point where the noise level is greatest.

which in turn is a modified version of the Navy Engine Performance Computer Program (NEPCOMP). ${ }^{13}$ The engine is represented as a one-dimensional flow path and thermodynamic conditions are computed at the entrance and exit of each component. Off-design performance is computed using input performance maps for the individual engine components.

ENGGEN also computes the weights and dimensions of each of the components of the engine. Once all the component dimensions have been calculated, they are assembled according to the engine architecture to form a layout of the complete engine geometry. The engine flow path geometry is output as a postscript file which serves as the starting point for further refinement of the geometry, as described in the next section.

\section{B. Geometry Post-Processing}

A geometry post-processing routine was created to process the simplified engine duct geometry from ENGGEN, enforce slope, curvature and wall intersection constraints, and modify the geometry based on additional design variables which could not be specified within ENGGEN. For example, ENGGEN only allows for a constant-area fan and the geometry output does not have the ability to extend the fan bypass duct beyond the minimum required length. To correct these and other shortcomings, the desired values for fan blade aspect ratio and contraction ratio are used to modify the duct in the vicinity of the fan, an inlet cone is added, the engine immediately behind the fan exit is extended to create a rotor-core stator spacing, a stator row is created with the desired aspect ratio, rotor-stator chord ratio and rotor-stator spacing, and the aft nacelle, fan nozzle, core cowl, core nozzle and plug are redrawn to enforce slope and curvature constraints on the outer nacelle.

\section{Fan Preliminary Design and Analysis}

For the more detailed fan noise analysis method used in this study it was necessary to obtain a realistic three-dimensional definition of the fan rotor and stator blades. To assure that the fan geometry was always matched to the engine cycle design, it was necessary incorporate a preliminary design methodology for the fan rotor and stator blades into the overall design and analysis process. This fan blade design process uses a two-step process which first determines the desired fan velocity triangles, then designs a rotor camber surface to produce the desired rotor outlet conditions with an appropriate chordwise and spanwise distribution of swirl. The two elements are described in the following sections.

\section{Fan Design Velocity Triangles}

The first step in the fan design process is to determine an appropriate spanwise distribution of rotor exit flow properties to ensure that the required fan pressure ratio is met while keeping the work required by each section of the blade span within a reasonable range. The streamline curvature code MERIDLN is used to analyze the flow through the fan rotor and stator rows. MERIDLN is an unpublished revision of MERIDL ${ }^{14}$ 


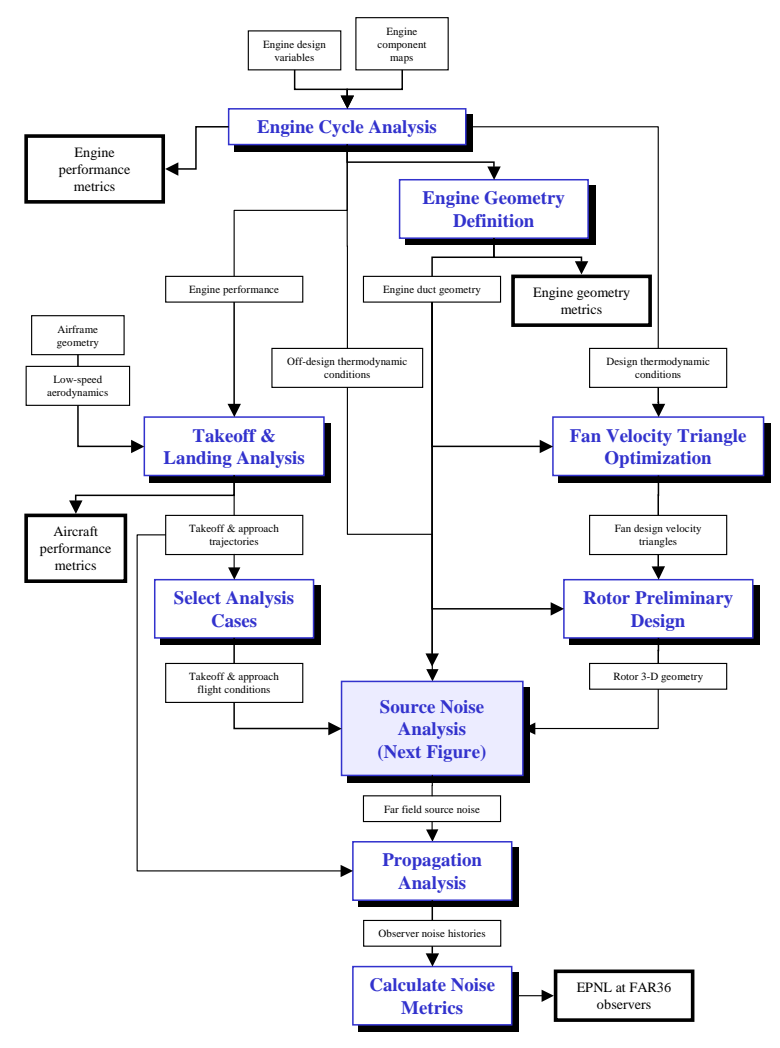

(a) full flow chart

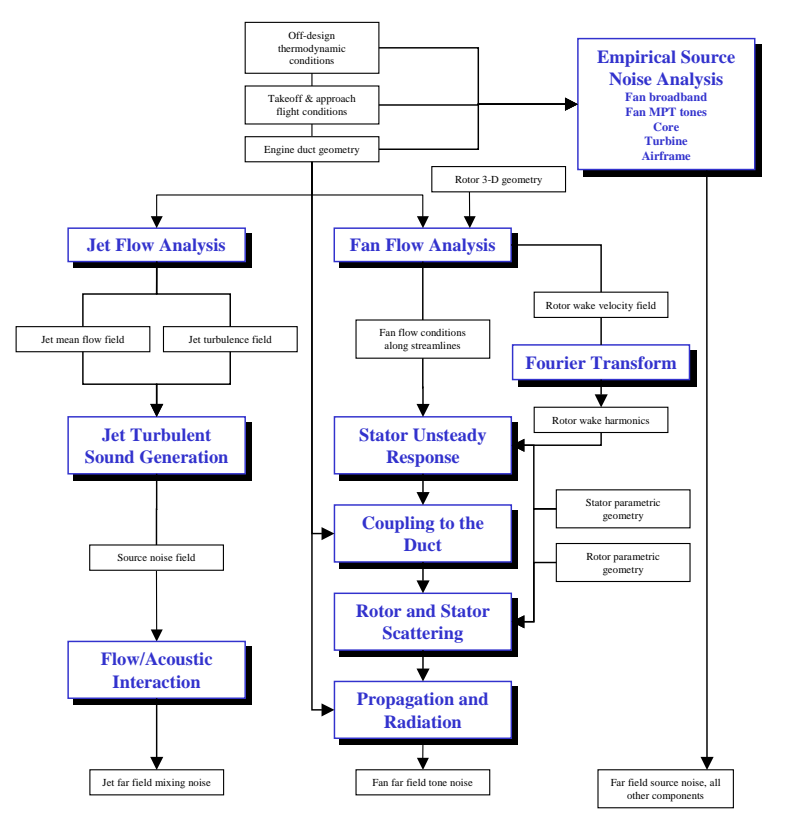

(b) detailed noise analysis block

Figure 2. Performance and noise analysis flow chart.

which obtains a detailed subsonic or shock-free transonic flow solution on the midchannel stream surface (meridional plane) of a turbomachine or annular duct. The distribution of tangential velocity is specified along a radial line downstream of each blade row, and the total pressure loss for each blade is input as a function of radius.

To find an optimum radial work distribution, MERIDLN analysis is used in combination with the CONMIN optimization routine. ${ }^{15}$ The design variables are the values of tangential velocity at a number of discrete radial locations, typically 6 to 10 values, from hub to tip at a location just aft of the rotor. The radial work profile is optimized by minimizing the objective function

$$
F=\sum_{i=1}^{n}\left(p_{r, i}-p_{r, d e s}\right)^{2}
$$

where $n$ is the number of streamlines, $p_{r, i}$ is the total pressure ratio along the $i^{t h}$ streamline as computed by MERIDLN and $p_{r, \text { des }}$ is the design fan pressure ratio. This formulation for the objective function seeks to minimize the radial variation in pressure coefficient, so a minimum value of zero would be achieved by a constant-work rotor design. The constraints are the maximum rotor and stator diffusion factors, rotor flow turning, rotor exit relative Mach number, stator inlet Mach number, and maximum and minimum rotor degree of reaction for each streamline, with the final two inequality constraints forming an acceptable bounding region on the fan pressure ratio.

\section{Rotor Geometric Design}

Once the design radial work distribution has been chosen using the procedure in the previous section, the fan rotor blade surface geometry is optimized through a three-dimensional inverse design procedure in combination with Swift. ${ }^{16,17,18,19}$ Swift is a multi-block computer version of the Rotor Viscous 3-D code (RVC3D $)^{20,21,22,23}$ and is used for computational fluid dynamics analysis of three-dimensional viscous or inviscid flows in turbomachinery. The program solves the thin-layer Navier-Stokes equations using an explicit 
finite-difference technique. The rotor computational grid used in Swift is generated using the companion TCGRID computer program, ${ }^{24}$ which is an elliptic grid generator originally intended for isolated airfoils, but has been modified to allow generation of periodic C-type grids for turbomachinery applications.

In the inverse design procedure, the goal is to arrive at a three-dimensional blade shape which will achieve a proper axial distribution of swirl, $r V_{\theta}(z)$, through the rotor at each radial station along the blade. An appropriate swirl distribution can be prescribed for the purpose of achieving the design pressure ratio while avoiding large rotor surface pressure gradients which could lead to separated flow.

Rather than prescribing the swirl distribution directly, though, it is more natural instead to start with a prescribed axial distribution for the static pressure differential between the upper and lower surfaces of the blade. An appropriate pressure differential $\Delta p(z)$ begins with a minimum (negative) value $\Delta p_{\text {min }}$ at the leading edge of the blade and remains nearly constant up to a shock location $z_{\text {shock}}$, followed by a discontinuous jump in pressure differential through the shock and then a gradual increase to zero at the trailing edge (Figure 3). The circumferential momentum equation

$$
\Delta p(z)=\dot{m} \frac{d \bar{V}_{\theta}}{d z}
$$

is then integrated to obtain the swirl schedule. For leading-edge Mach numbers less than 1.3, values of $z_{\text {shock }}=0.3$ and $F_{\text {shock }}=1$ are used to defined a controlled-diffusion or supercritical blade section, and for leading-edge Mach numbers greater than 1.3, the shock strength is an empirical function of the leading-edge Mach number.

Once the swirl distribution has been prescribed, it can be used to define the required axial and radial distributions of the blade camber. The blade camber surface is defined by a blade shape function $f(r, z)$ which is the tangential coordinate of the blade camber line in radians. For a thin blade the circumferentially-averaged tangential velocity is approximately tangent to the blade camber surface, so an initial camber surface can be defined by enforcing the flow-tangency condition along the blade surface:

$$
V_{r} \frac{\partial f}{\partial r}+V_{z} \frac{\partial f}{\partial z}=\frac{V_{\theta}^{*}}{r}-\Omega
$$

where $V_{z}$ and $V_{r}$ are the axial and radial components of velocity and $\Omega$ is the blade rotational speed. ${ }^{27}$

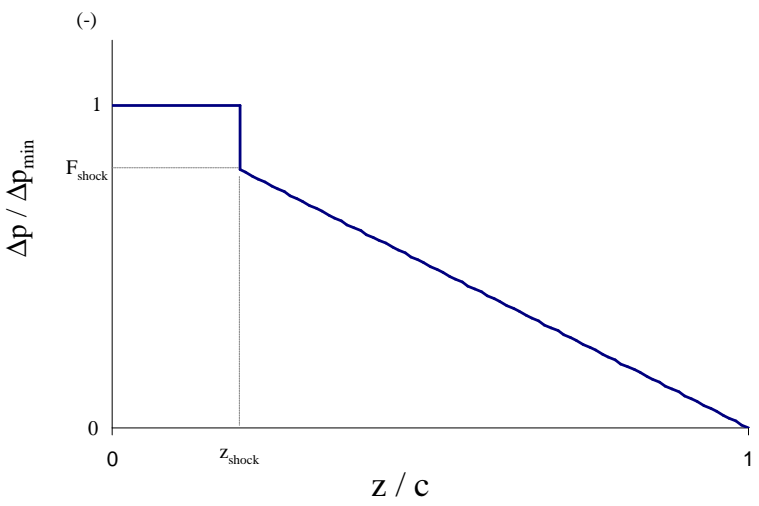

Figure 3. Prescribed blade pressure loading. The tangential velocity is starred to indicate that it is a prescribed distribution. The flow field through the blade is not initially known, however, so initial estimates of the velocity components must be used, and the camber surface must be refined through iteration to find the surface which produces a swirl distribution exactly equal to the prescribed one. Of course, the circumferentially-averaged tangential velocity is only approximately tangent to the blade camber surface, so it also is necessary to correct the prescribed swirl distribution during each iteration to account for the actual difference between the circumferentially-averaged and camber line tangential velocities.

\section{Flight Performance}

Using the computed engine performance and input aircraft low-speed aerodynamics and weight information, the takeoff and landing field lengths and flight trajectories are computed by the takeoff and landing module of the Flight Optimization System (FLOPS) computer program. ${ }^{28}$ FLOPS is a multidisciplinary suite of computer routines for mission analysis and optimization of aircraft, which are integrated in a monolithic structure. The takeoff and landing module computes detailed performance of the aircraft using time-integrated solution of the equations of motion. The analysis is carried out while obeying all relevant FAR 25 rules $^{29}$ regarding engine-out takeoff and aborted takeoff, all-engine aborted takeoff, engine-out aborted landing, and minimum first-segment, second-segment, and missed-approach available engine-out climb gradients.

A new utility was created to carry out the interpolation of the engine operating conditions and flight conditions at the sideline, cutback and approach flight conditions. The routine examines the takeoff and 
approach flight paths that are output from FLOPS and locates the points in the takeoff flight path at which the aircraft passes through the defined sideline altitude and the defined cutback distance, and the point in the approach flight path at which the aircraft descends through the defined approach altitude (Figure 4). For each of these points, the flight conditions for noise analysis are interpolated from the takeoff or landing flight path.

\section{E. Noise Analysis}

Two physics-based noise analysis methods were chosen for use in this study: a method for computation of fan rotor-stator interaction tones, and a method for jet turbulent mixing noise. These methods are discussed in detail in the following sections. To keep the problem tractable it was necessary to limit the use of physics-based methods to just the fan tone noise and jet mixing noise, which are two of the most important sources to be considered. All additional sources, including fan broadband and multiple pure tone noise, core noise, turbine noise, jet shock cell

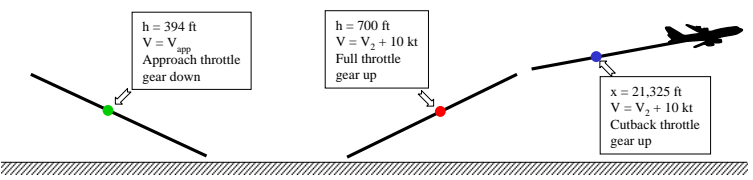
noise, and airframe noise, as well as the effects of fan inlet and exhaust duct acoustic treatment, were computed using empirical methods.

\section{Fan Flow Field}

The fan interaction tone noise analysis involves a two-step process: first, the fan flow field is computed using computational fluid dynamics (CFD) analysis, then the flow field information is used to calculate the acoustic response of the stator. The rotor flow field and downstream wake profiles are computed using Swift, which was described previously in Section III.C.2. The rotor grid is generated using TCGRID with the outlet plane of the grid located at the leading edge of the stator to capture the rotor wake properties. Once convergence has been achieved, a post-processor reads the computed three-dimensional flow field, extracts the wake velocities at the stator leading edge, and performs circumferential averaging of the flow field properties to arrive at the coordinates of the streamlines and the velocity components and thermodynamic properties along the streamlines.

\section{Fan Rotor-Stator Tone Noise}

Once the fan flow field and blade wake shapes have been determined, the fan rotor/stator interaction tone noise and coupling to the duct is computed using the Tone Fan Noise Design/Prediction System (TFaNS). ${ }^{30,31}$ TFaNS is a loosely-coupled set of computer programs for computing the tone noise at the far field for a number of BPF harmonics. TFaNS computes the tones generated from the interactions of the rotor blade wakes with the stator vanes using a simplified fan geometry with a constant-area annular duct and flat plate stators. The program determines the coupling of the generated tones with the duct modes in the immediate vicinity of the stator row, assuming mean axial flow. Flat-plate models are also used to calculate the scattering of upstream and downstream propagating modes by both the rotor and stator. The propagation of the modes through the duct and their eventual radiation to the far field is calculated using inlet and exhaust finite element radiation codes. ${ }^{32,33}$ Finally, the results for the rotor/stator interaction source noise, rotor and stator scattering coefficients, and radiation calculations are combined into a coupled system to account for the reflection and transmission of acoustic and vorticity waves in both the inlet and aft directions.

\section{Jet Flow Field}

A two-stage methodology is also used to compute the jet mixing noise. First, aerodynamic calculations of the time-averaged turbulent flow field are carried out using CFD analysis, and the computed time-averaged mean flow and turbulence properties are used for the noise calculations. Computation of the flow field is carried out using the WIND flow solver ${ }^{34}$ to solve the Thin-layer Navier-Stokes equations in conservation law form. The second-order upwind-biased Roe differencing is used, and the gas is treated as a thermally 
perfect gas. A two-equation shear stress transport (SST) turbulence model is used to initialize the flow field, after which the Chien $\mathrm{k}-\epsilon$ model is used to complete the convergence. Three levels of grid sequencing are used to accelerate convergence of the solution.

A suitable axisymmetric computational grid for the aft engine geometry and downstream flow field is generated using a new algebraic grid generation method. The grid spacing is stretched uniformly in the axial direction to allow clustering of grid points at each nozzle exit and at the plug tip. The grid is also stretched in the radial direction to allow clustering at each duct wall and in the two shear layers downstream of the nozzle exit planes. The radial stretching is constant within each duct, then is relaxed exponentially downstream of each nozzle exit to allow the clustering to be expanded as the shear layers grow.

\section{Jet Mixing Noise}

Jet mixing noise is computed using the MGBK computer program, ${ }^{35}$ which is a modified version of the original MGB program. ${ }^{36}$ From the mean flow properties and the $k$ and $\epsilon$ fields calculated in the CFD analysis, MGBK uses the Lighthill-Ribner method to predict the source noise generated by turbulent fluctuations in the mixing regions, and Lilley's equation for sound/flow interaction and propagation to the far field. The program uses two empirical factors, $\alpha_{c}$ and $\beta_{c}$, to compute a modified directivity function to account for the effect of the aircraft's forward speed.

\section{Additional Noise Sources}

The additional sources, including fan broadband and multiple pure tone noise, core noise, turbine noise, jet shock cell noise, airframe noise, and fan inlet and exhaust duct treatment suppression, are computed using the empirical methods in ANOPP, which is described in the next section. The modularity of the methodology, though, assures that additional physics-base methods for these other noise sources could be integrated into the methodology in the future without having to restructure the entire process.

\section{Far-Field Propagation}

Output from the detailed noise analysis block (Figure 2(b)) consists of the total fan rotor-stator interaction tone and jet mixing far field noise spectra, as a function of directivity angle, for each of the three flight conditions, plus results of the empirical analysis for all other noise sources. The far field source noise levels are passed to the Aircraft Noise Prediction Program (ANOPP) ${ }^{37,38}$ for propagation to the sideline, cutback and approach observer locations. ANOPP is a computer program for the prediction of aircraft noise levels using empirical methods for the various engine and airframe noise sources, and simplified physical models for propagation, atmospheric attenuation and ground impedance. The EPNL metric at each observer location is computed from the time history of the noise spectra at the observer.

\section{Implementation}

The analysis process that was assembled in this study accelerates the evolution of an aircraft and engine concept from conceptual-level definition of engine geometry and operating conditions to a preliminary-level analysis of the noise levels. Execution of the methodology still requires approximately one day per design point, however, so it was necessary to take additional steps in the implementation of the methodology before it became practical for use as a conceptual design tool.

\section{A. Response Surface Methodology}

One way to increase the utility of high-fidelity analysis methods is through the use of surrogate-based optimization, which uses fast-executing surrogate models in place of the high-fidelity analysis when conducting optimization problems that require large numbers of function evaluations. Among all categories of surrogate models, global approximation methods are of particular interest in conceptual design, because once created they can be used in any number of studies to explore the full domain of possible designs without the need to return repeatedly to the high-fidelity methods for additional analysis. Global approximation methods seek to create a metamodel which retains the overall properties of the complex analysis, but can be executed much more quickly. ${ }^{39}$ In general, metamodels are created by executing the analysis method for a limited 
number of points distributed throughout the design space, and the results are used to infer relationships between the design variables and the analysis outputs which can be used to obtain the approximated value at any point in the design space.

In this study, response surface methodology (RSM) was used in the creation of metamodels. RSM is composed of a number of statistical techniques for empirically relating an output variable, or response, to the values of several selected input variables. ${ }^{40}$ The relationship is specified as a response surface equation (RSE), which is an algebraic function, usually polynomial, for the response as a function of the input variables. A second-degree polynomial RSE has the following form:

$$
R=b_{0}+\sum_{i=1}^{n} b_{i} x_{i}+\sum_{i=1}^{n} b_{i i} x_{i}^{2}+\sum_{i=1}^{n-1} \sum_{j=i+1}^{n} b_{i j} x_{i} x_{j}
$$

where $b_{0}$ is the intercept, $b_{i}$ are regression coefficients for the linear terms, $b_{i i}$ are regression coefficients for the pure quadratic terms, and $b_{i j}$ are regression coefficients for the cross-product terms. The $x_{i}$ variables represent normalized values of each of the input variables, or factors, affecting the response. The seconddegree model allows for both linear and non-linear behavior in individual factors, as well as simple interactions between factors. An illustration of a simple two-variable RSE is shown in Figure 5.

\section{Design of Experiments}

The most common method of obtaining the regression coefficients in the RSE is through design of experiments (DOE), which provides an efficient and methodical system for determining the necessary combinations of factor levels for obtaining the maximum regression information with a minimum number of runs. ${ }^{41}$ The specified combinations of factors are organized into a DOE table, whose rows represent a number of analysis runs and whose columns represent the specified factor combinations for each of those runs. For example, Table 1 represents a simple two-level, full-factorial DOE table for three design variables: $x_{1}, x_{2}$, and $x_{3}$. Each of the rows in the table represents one of eight analysis runs, while the first three columns represent the values of the three factors and the final column is used to record the output values

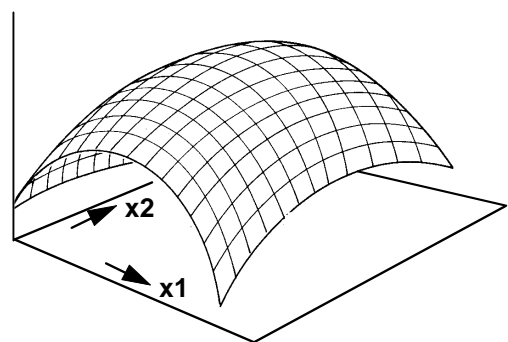

Figure 5. Illustration of a twovariable RSE. from each of the analysis runs. The variable ranges are normalized so the minimum value corresponds to -1 , while the maximum value corresponds to +1 . Normalization of the factors in this manner serves to reduce the potential numerical error during calculation of the RSE coefficients and simplifies the mathematics.

Table 1. Example two-level full-factorial DOE table for three variables.

\begin{tabular}{c|ccc|c} 
& \multicolumn{3}{|c|}{ Factors } & Response \\
Run & $\overline{x_{1}}$ & $\overline{x_{2}}$ & $\overline{x_{3}}$ & $\mathbf{y}$ \\
\hline $\mathbf{1}$ & -1 & -1 & -1 & $y_{1}$ \\
$\mathbf{2}$ & +1 & -1 & -1 & $y_{2}$ \\
$\mathbf{3}$ & -1 & +1 & -1 & $y_{3}$ \\
$\mathbf{4}$ & +1 & +1 & -1 & $y_{4}$ \\
$\mathbf{5}$ & -1 & -1 & +1 & $y_{5}$ \\
$\mathbf{6}$ & +1 & -1 & +1 & $y_{6}$ \\
$\mathbf{7}$ & -1 & +1 & +1 & $y_{7}$ \\
$\mathbf{8}$ & +1 & +1 & +1 & $y_{8}$
\end{tabular}

\section{Variable Screening}

Analysis outputs for a complex system such as an aircraft can be affected by a very large number of different variables. As the number of factors is increased, the number of runs can quickly become impractical for 
the quadratic model, even when using an efficient design. To reduce the number of variables required to a manageable level, it is often necessary to conduct a screening study: first, a two-level DOE is used to estimate the linear terms in the RSE, and the results analyzed to determine which variables have the largest effect on the variation of the response. Only the most important variables are retained for development of the final quadratic equation using a DOE table with three or more levels. In this way, the variables which do not contribute significantly to the variation of the response are eliminated, greatly reducing the number of runs without significantly degrading the accuracy of the metamodel.

\section{Creation of RSEs}

Creation of a quadratic RSE requires DOE design types with three or more levels. One useful category of designs is central-composite designs (CCD), which use a two-level full- or fraction-factorial design, augmented by a center point and a series of "star points" along each variable axis. Figure 6 shows a plot of the factor combinations for a sample three-variable CCD. When the star points are located at \pm 1 on each axis, a special class of CCD is created, called a face-centered design. Face-centered CCDs are especially useful when the outputs are the result of computational simulation rather than actual experimental measurements.

Another category of designs which is useful for generation of RSEs is D-optimal designs, which can be used in cases where the design space is irregular, where a nonstandard model is desired, or when the number of sample sizes needs to be kept as small as possible. Creating a D-optimal design involves finding, for the specified form of response function and number of runs, an appropriate design matrix which gives the best precision in the estimation of the RSE coefficients. The Doptimal design is the value of the design matrix, $\mathbf{X}$, which minimizes the $D$ criterion:

$$
D=\left|\left(\mathbf{X}^{\prime} \mathbf{X}\right)^{-1}\right|
$$

If the number of candidate feasible designs is large, finding the optimum design is not feasible, but a numerical optimizer can be used to find a design table with a sufficiently low value of $D .{ }^{42,43}$



Figure 6. Three-variable central composite design.

\section{B. Probabilistic Analysis}

To properly assess the risk of failing to meet the design constraints or performance and noise targets, it is necessary to accurately predict the probability distributions for each of the design metrics using probabilistic methods. The method used in this research was Monte Carlo analysis, in which sample points are generated by selecting random samples from probability distributions of the input variables. The implementation of Monte Carlo analysis is simple since the analysis methods can be used directly and no gradient calculations or iteration are needed, the level of accuracy can be controlled through the use of greater numbers of simulations, and any number of input variables can be used. However, Monte Carlo analysis is not practical if the analysis cannot be performed quickly because it can require thousands of analysis runs to accurately calculate the probability density function. When used in combination with metamodels, however, Monte Carlo analysis becomes practical to use, but with the disadvantage that the problem becomes subject to the limitations on numbers of variables and accuracy that are inherent to the metamodels.

\section{Results}

\section{A. Baseline Definition}

To make the problem tractable, the study was limited to a single class of aircraft and engine, though in the design and risk analysis studies the major design variables - such as gross weight, bypass ratio, etc. - were varied significantly relative to the baseline values. Figure 7 shows the baseline aircraft used in the study, and Table 2 gives the major design variables. The aircraft is based on the NASA Inter-center Systems Analysis Team 300-passenger twin-engine baseline, with a maximum gross weight of 600,000 pounds and a nominal design range of 6500 nautical miles, and is similar in size and mission to a Boeing 777 . The baseline is powered by a separate-flow, two-spool high-bypass turbofan engine with a baseline thrust of 90,000 pounds 
and a bypass ratio of 8.5, which is similar to a General Electric GE90 engine. Figure 8 shows the calculated noise levels at each observer for the baseline aircraft and engine, broken down by component.

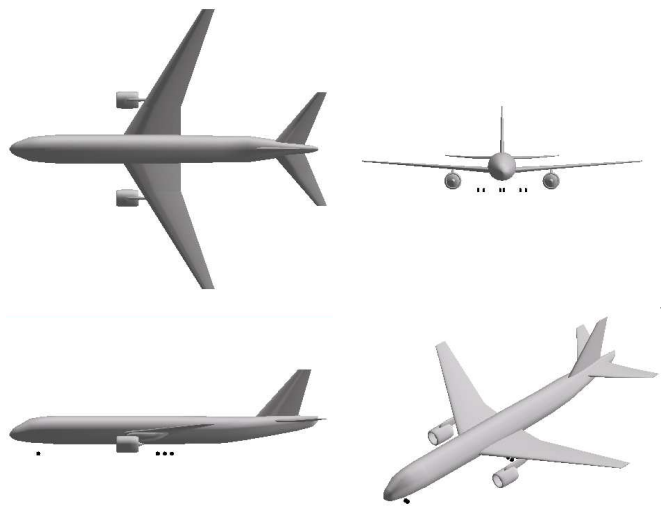

(a) baseline airframe

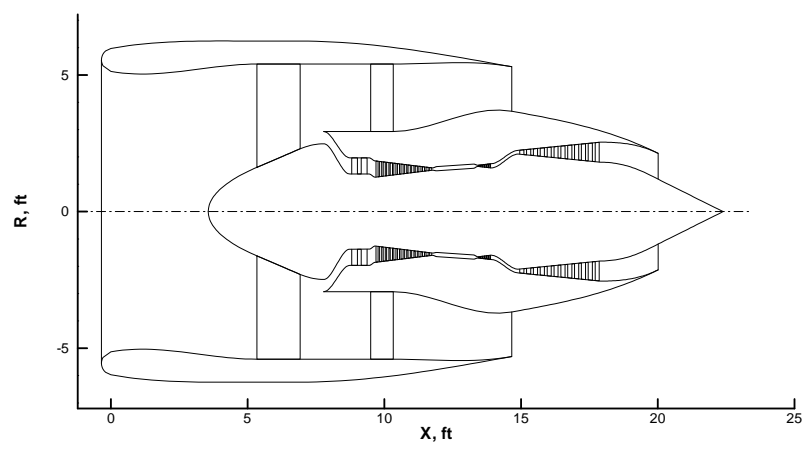

(b) baseline engine

Figure 7. Baseline aircraft and engine.

Table 2. Baseline aircraft and engine design variables.

\begin{tabular}{c|c} 
Baseline aircraft & Baseline engine \\
\hline Passengers: 300 & Low-speed fan \\
Range: $6500 \mathrm{nmi}$ & Thrust: $90,000 \mathrm{lb}$ \\
Gross weight: $600,000 \mathrm{lb}$ & Bypass ratio: 8.5 \\
Wing area: $4600 \mathrm{ft}^{2}$ & Fan pressure ratio: 1.5 \\
Wing aspect ratio: 8.7 & Overall pressure ratio: 38 \\
Fuselage length: $220 \mathrm{ft}$ & Turbine inlet temperature: $3285 \mathrm{R}$
\end{tabular}

\section{B. Design and Uncertain Variables}

As a first step in conducting design studies of interest, a complete catalog of the major design variables was compiled. These variables fall into two categories: design variables, whose values can be chosen by the engine or airframe designer or at least can be known with reasonable accuracy in the early stages of the design process, and uncertain variables, whose values are not accurately known or can vary appreciably based on the operating environment. The complete list of variables is broken down below into engine design variables, airframe design variables, and uncertain variables; these three groups will be discussed in detail in the following sections.

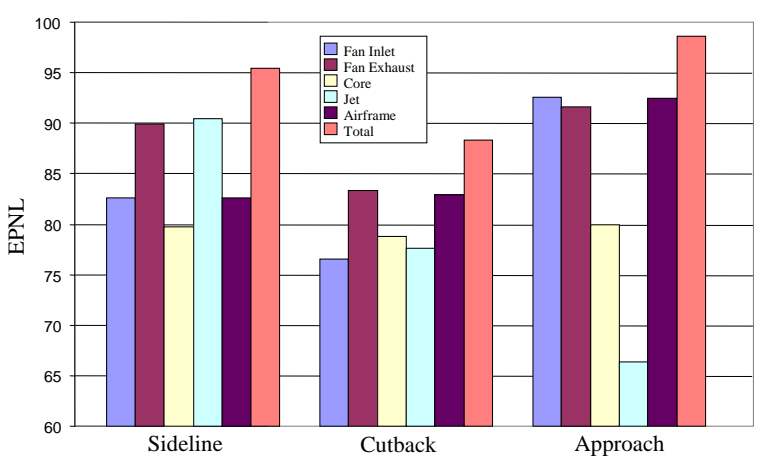

\section{Engine Design Variables}

Figure 8. Baseline noise levels at FAR 36 certification points.

A list of system-level engine design variables is given in Table 3. Most of these variables are inputs to ENGGEN, while some are used to refine the engine geometry. Minimum and maximum values are given for each variable, indicating the desired range within which each variable was allowed to vary in this study to affect the design. The ranges for the bypass ratio and overall pressure ratio were chosen to represent modest, non-revolutionary changes to the baseline engine, while the ranges for the other engine cycle design 
variables were chosen to ensure that the optimum cycle would be expected to fall within the design space for any value of bypass ratio or overall pressure ratio.

Table 3. Engine design variables and ranges.

\begin{tabular}{l|crr} 
Variable & Variable Name & Min & Max \\
\hline Bypass ratio & BPRDES & 8 & 11 \\
Fan pressure ratio & FPRDES & 1.3 & 1.5 \\
Overall pressure ratio & OPRDES & 30 & 50 \\
HPC press. ratio & HPCPR & 20 & 25 \\
Fan tip rotational speed, ft/sec & UTIP1 & 1000 & 1200 \\
Fan/turbine gear ratio & GRATIO & 1 & 3 \\
Fan hub/tip ratio & RH2T1 & 0.3 & 0.4 \\
Number of fan blades & NB & 20 & 24 \\
Fan rotor-stator spacing & RSSF & 1.5 & 2.0 \\
Fan stator sweep, deg & SSWP & 0 & 20
\end{tabular}

\section{Airframe Design Variables}

A list of airframe variables is given in Table 4. The aerodynamic design variables are derived by representing the takeoff and landing aerodynamics with lift-curve and drag polar equations of the following form:

$$
\begin{aligned}
C_{L} & =C_{L, 0}+C_{L, \alpha} \alpha \\
C_{D} & =C_{D, 0}+K_{1} C_{L}+K_{2} C_{L}^{2}
\end{aligned}
$$

where $C_{L}$ and $C_{D}$ are the lift and drag coefficients, respectively, at angle of attack $\alpha$. The coefficients $C_{L, 0}$, $C_{L, \alpha}, C_{D, 0}, K_{1}$ and $K_{2}$ can be varied to represent virtually any set of low-speed aerodynamics using just five variables for takeoff and five for landing. To further narrow the required list of aerodynamic variables, the coefficients were expressed as increments relative to the baseline values, with the increments applying to both the takeoff and landing polars.

\section{Uncertain Variables}

A list of uncertain variables was compiled by examining the input variables for all the computer programs used in the analysis process and determining which variables could be expected to be different from their defaults due to lack of knowledge about the engine and aircraft at the conceptual design stage. The complete list of uncertain variables is shown in Table 5 . The list includes engine component efficiencies and mass flow and power bleed requirements, aircraft takeoff and landing operating conditions and performance, and allowances for modelling errors for the individual noise components.

\section{Design Space Limitations}

Ideally, the design space for a DOE would be a hypercube in which all of the design variables were allowed to vary between their minimum and maximum values regardless of the values of all other variables. In reality, however, there are combinations of design variables which result in designs which are infeasible either because they violate physical laws or because it is impossible for the design to meet all its constraints or to perform at all required operating conditions. Aircraft engines in particular can encounter these types of limitations on the design space.

An extensive study was conducted to find the combinations of design variable values under which the engine cycle design or the fan velocity triangle design methods fail. The findings were then used to define a set of constraints defining the combinations of values which exist within the feasible region of the design space. Unfortunately, the combination of constraints resulted in elimination of approximately half of the original design space. The restriction of the design space had important implications regarding the choice of DOE tables used to create the RSEs and ultimately proved to have a significant impact on the accuracy of the analysis results, as will be seen in later sections. 
Table 4. Airframe variables and ranges.

\begin{tabular}{l|crr} 
Variable & Variable Name & Min & Max \\
\hline Aircraft thrust/weight ratio & TWRAT & 0.24 & 0.30 \\
Wing loading, lb/sq. ft. & SW & 120 & 140 \\
Wing aspect ratio & AR & 8 & 9 \\
Flap area ratio & FLAPR & 0.25 & 0.35 \\
Horizontal tail area, sq. ft. & SHT & 1000 & 1500 \\
Vertical tail area, sq. ft. & SVT & 600 & 900 \\
$\Delta C_{L, 0}$ & DCL0 & -0.05 & 0.05 \\
$\Delta C_{L, \alpha}$, deg $^{-1}$ & DCLALF & -0.01 & 0.01 \\
$\Delta C_{D, 0}$ & DCD0 & -0.011 & 0.011 \\
$\Delta K_{1}$ & DZK1 & -0.004 & 0.004 \\
$\Delta K_{2}$ & DZK2 & -0.005 & 0.005 \\
$\Delta C_{L, m a x}$ & DCLMAX & -0.25 & 0.25 \\
Number of main gear trucks & NMG & 2 & 4 \\
Number of nose gear trucks & NNG & 1 & 2 \\
Number of wheels per main truck & NWMG & 2 & 4 \\
Number of wheels per nose truck & NWNG & 2 & 4 \\
Main gear wheel diameter, ft & DMG & 2.5 & 4 \\
Nose gear wheel diameter, ft & DNG & 2.5 & 4 \\
Number of flap slots & NSA & 1 & 3 \\
Thrust inclination on the ground, deg & TINC & 0 & 2 \\
Angle of attack on the ground, deg & ALPRUN & -1 & 2
\end{tabular}

\section{Variable Screening}

A screening study was conducted to determine which of the engine design variables, airframe variables and uncertain variables in Tables 3-5 had the largest effect on the different metrics, and to arrive at a subset of variables which should be used in developing metamodels for design studies. The metrics that were calculated were the sideline, cutback and approach EPNLs, fan diameter, design core nozzle pressure ratio (NPR), takeoff and landing field lengths and the approach velocity. Two-level fractional-factorial DOE tables were created and the analysis process was executed for each of the design variable combinations.

\section{Development of Response Surface Equations}

Using the results of the screening studies as guidance, a final list of variables was selected for use in development of RSEs. To limit the total analysis time required to create the RSEs to approximately two months, it was necessary to limit the number of high-fidelity analyses to approximately 40 cases. Consequently, six variables were chosen from the engine variables and two from the flight path variables, and a set of 40-run D-optimal designs was created for each of the noise metrics. The D-optimal designs were constrained to the feasible design space discussed in Section V.B.4, and to limit the number of terms in the RSE to fewer than 40, the interaction terms between the engine variables and the flight path variables were neglected. Separately, a three-variable, 15-run face-centered CCD was created to handle three propagation variables, and the two DOE tables were crossed with each other to create a large 600-run hybrid DOE. Face-centered CCDs were used for the takeoff and landing field lengths and a full-factorial design for approach velocity.

After the analysis cases from the DOE table were run, the results were analyzed statistically to obtain the coefficients for the RSEs. Parametric sensitivity plots for the three noise levels are shown in Figure 9, with two additional rows showing the sensitivies for the two most dominant noise sources: fan and jet noise for sideline and cutback, and fan and airframe noise for approach. Parametric sensitivity plots for the performance and geometry metrics are shown in Figure 10. These plots show the relationship between each of the individual variables and the noise level when all other variables values are kept constant at their 
Table 5. Uncertain variables and ranges.

\begin{tabular}{l|crr} 
Variable & Variable Name & Min & Max \\
\hline$\Delta$ Fan efficiency & DEFFFAN & -0.005 & 0.005 \\
$\Delta$ HPC efficiency & DEFFHPC & -0.005 & 0.005 \\
$\Delta$ Burner efficiency & DEFFBURN & -0.005 & 0.005 \\
$\Delta$ Turbine efficiency & DEFFTUR & -0.005 & 0.005 \\
$\Delta$ Primary nozzle thrust coefficient & DEFFNOZ1 & -0.005 & 0.005 \\
$\Delta$ Fan nozzle thrust coefficient & DEFFNOZ2 & -0.005 & 0.005 \\
Turbine cooling mass flow / HPC mass flow & WCOOL & 0.25 & 0.30 \\
Customer power extraction, Hp & HPEXT & 0 & 100 \\
Customer bleed, lb/sec & COSTBL & 3 & 5 \\
Rolling friction coefficient & ROLLMU & 0.023 & 0.027 \\
Braking friction coefficient & BRAKMU & 0.25 & 0.35 \\
Aspect ratio for ground effect / AR & ARGEF & 0.8 & 1.2 \\
Engine cutback rate & SPRATE & 0.05 & 0.15 \\
Aircraft rotation rate, deg/sec & VANGL & 1.5 & 2.5 \\
Braking delay after touchdown, sec & TIBRAK & 2 & 5 \\
Pilot reaction time after engine failure, sec & PILOTT & 1 & 3 \\
Gear drag coefficient & CDGEAR & 0.015 & 0.025 \\
Engine out drag coefficient & CDEOUT & 0.001 & 0.002 \\
Ground specific flow resistance & SIGMA & 450 & 500 \\
Fan inlet noise modelling error, dB & -SUPPLI & -3 & 3 \\
Fan aft noise modelling error, dB & -SUPPLE & -3 & 3 \\
Core noise modelling error, dB & -SUPPLC & -4 & 4 \\
Turbine noise modelling error, dB & -SUPPLT & -4 & 4 \\
Jet noise modelling error, dB & -SUPPLJ & -2 & 2 \\
Airframe noise modelling error, dB & -SUPPLR & -5 & 5
\end{tabular}

midpoints; they help to demonstrate the general trend as the individual variable is varied, but do not show the interactions that exist between the variables which are nonetheless captured by the RSEs.

\section{E. Validation of RSEs}

Table 6 gives diagnostics metrics for each of the RSEs to give an indication of the quality of the fit. The first two columns indicate how well the RSEs fit the points from the DOE table, while the final three columns indicate how well the RSEs fit an additional set of points randomly selected from the design space. For the fitting cases, the RMS error is the root-mean-square value of the differences between the RSE output and the actual value, and quantifies the error inherent in approximating the function with a second-degree quadratic equation. The coefficient of multiple determination, or $R^{2}$, measures the mean-square error normalized by the total variation of the RSE, so that a value of unity indicates a perfect fit. The worst fit is exhibited by the approach EPNL, which has an $R^{2}$ of only 0.87 and an RMS error of greater than 1 EPNdB. The poor fit might be due to the omission of interaction terms between the engine and flight path variables, which do not seem to be important for sideline and cutback noise but might be more important at approach, or it might be a result of spurious correlations in the DOE table resulting from the severe restrictions on the design space. The fitting errors in the takeoff field length RSE are mostly like due to the fact that takeoff field length is affected by a large set of variables with three-variable and higher interactions. In contrast, the core NPR fit is nearly perfect because the core nozzle exit pressure is affected by only a handful of variables, and within the current design space the behavior is nearly linear.

For the validation cases, the first column is the arithmetic mean of the errors at all validation points, and shows whether the errors are biased toward under-prediction or over-prediction; the second column shows the 


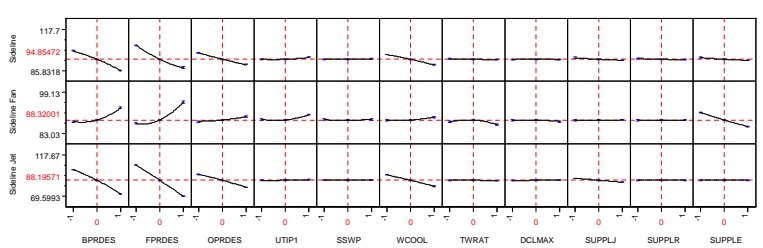

(a) sideline EPNL

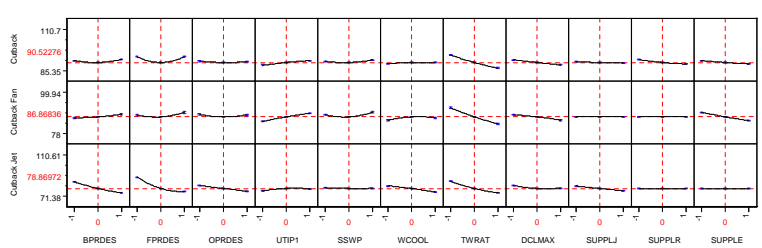

(b) cutback EPNL

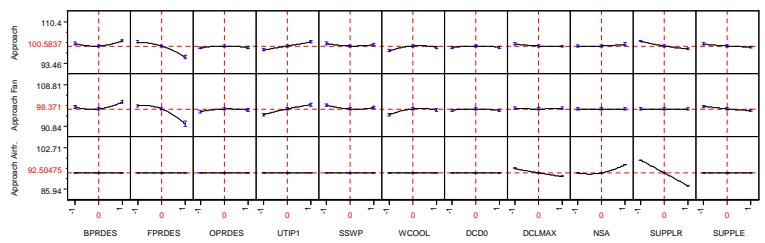

(c) approach EPNL

Figure 9. Parametric sensitivity plots for sideline, cutback and approach noise levels.

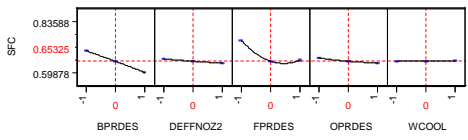

(a) cruise SFC

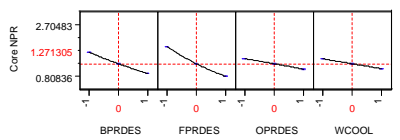

(b) core NPR

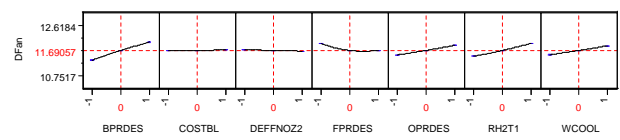

(c) fan diameter

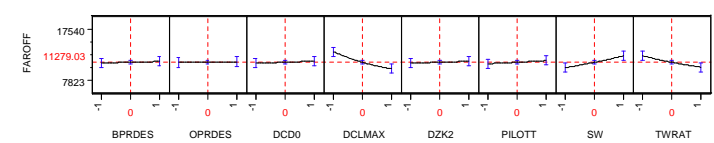

(d) takeoff field length

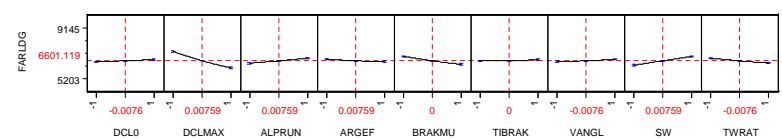

(e) landing field length

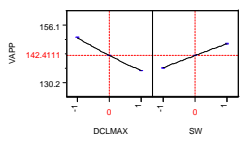

(f) approach velocity

Figure 10. Parametric sensitivity plots for geometric and performance constraints. 
mean approximation error which can be expected whenever the RSE is used to give an approximated value; and the third column gives the maximum absolute error encountered at any of the design points examined.

Table 6. Quality of fit metrics for RSEs.

\begin{tabular}{l|cc|ccc}
\multirow{2}{*}{ Metric } & \multicolumn{2}{|c}{ Fitting cases } & \multicolumn{3}{c}{ Validation cases } \\
\hline SFC & RMS error & $R^{2}$ & Mean error & RMS error & Max. error \\
Core NPR & 0.008122 & 0.9898 & $-0.86 \%$ & $3.96 \%$ & $16.56 \%$ \\
Fan diameter & 0.004259 & 0.9999 & $0.11 \%$ & $0.70 \%$ & $2.06 \%$ \\
Takeoff field length & $0.006539 \mathrm{ft}$ & 0.9997 & $-0.04 \%$ & $0.43 \%$ & $1.90 \%$ \\
Landing field length & $628.3 \mathrm{ft}$ & 0.9380 & $-0.35 \%$ & $4.48 \%$ & $15.99 \%$ \\
Approach velocity & $39.79 \mathrm{ft}$ & 0.9980 & $0.34 \%$ & $0.90 \%$ & $3.86 \%$ \\
Sideline EPNL & $0.3768 \mathrm{kt}$ & 0.9986 & $-0.09 \%$ & $0.18 \%$ & $0.53 \%$ \\
Cutback EPNL & $0.7149 \mathrm{EPNdB}$ & 0.9913 & $-0.35 \mathrm{EPNdB}$ & $1.57 \mathrm{EPNdB}$ & $4.09 \mathrm{EPNdB}$ \\
Approach EPNL & $0.6528 \mathrm{EPNdB}$ & 0.9844 & $-0.10 \mathrm{EPNdB}$ & $1.16 \mathrm{EPNdB}$ & $2.05 \mathrm{EPNdB}$
\end{tabular}

Even though the RSE for the cruise SFC does a good job of fitting the points in the original DOE table, the errors are significant when the RSE is used to predict SFC for other design points. Much of the errors are probably due to the lack of orthogonality in the D-optimal design table resulting from the considerable restrictions on the bounds of the feasible design space. Additional errors could arise if the SFC behaves in a more complex manner than a second-degree polynomial can capture. The approximation errors for takeoff field length appear to be due mostly to the lack of fit in the RSE, as evidenced by the fact that the RMS model-fitting error and the RMS approximation error are comparable.

\section{Analysis and Discussion}

Once RSEs were created to give rapid estimates of the performance and noise metrics as functions of the design variables, design studies could be performed to study the tradeoffs between performance and noise considerations, to quantify the effects of uncertainty, and to assess the benefits of swept stators as a noise-reduction technology. Since the metamodels could be executed rapidly, design studies could be carried out which involved many thousands of analyses but could still be performed in a timely manner while still capturing the results of the physics-based analysis of the fan and jet noise levels.

\section{A. SFC vs. Noise}

If noise were not an issue, the engine would normally be designed to minimize the mission fuel while meeting all constraints such as engine dimensions, flight envelope, aeromechanical design limits, and engine and airframe airworthiness requirements. Since analysis of the full aircraft mission is outside the scope of the current analysis process, the cruise point SFC is used as a surrogate for the total mission fuel since the latter is normally minimized at or near the minimum for the former. The aircraft's noise levels are combined into a cumulative noise level, which is the sum of the sideline, cutback and approach EPNLs minus the sum of the FAR 36 Stage 3 sideline, cutback and approach noise limits, and gives a single metric with which to rate the noise produced by the aircraft; a cumulative noise level of -10 EPNdB corresponds to Chapter 4 noise limits, assuming that the other criteria are met.

Figure 11 illustrates the tradeoff between cruise SFC and the cumulative noise level under various constraint scenarios. Each of the solid lines plots the locus of points having the minimum SFC for a given cumulative noise level. The first line shows the trend line when the core NPR and fan diameter are unconstrained, and the engine cycle is constrained only by the boundaries of the design space. Not surprisingly, the minimum SFC lies along the edge of the design space at the maximum allowable bypass ratio (BPR). Without constraints, there is no tradeoff between SFC and noise, and the two metrics decrease together without limit.

Realistically, though, the SFC and cumulative noise cannot continue to be reduced together because the amount of work required to drive the fan and compressors eventually exceeds the amount of work available 
from the turbines. The second line shows the trend line with a core NPR constraint imposed, forcing the engine to adhere to the work constraints of the turbine. Activation of the constraint imposes a minimum on the SFC, and any additional reduction in noise can only be achieved by reducing the BPR and simultaneously increasing the fan pressure ratio $(\mathrm{FPR})$ and overall pressure ratio $(\mathrm{OPR})$ to avoid violating the constraint, resulting in an SFC penalty which grows larger as the noise level continues to decrease. The portion of this line to the left of the absolute minimum SFC represents a Pareto set, which is the set of all Pareto optimal points, i.e. points at which the cumulative noise margin cannot be reduced without increasing the SFC, or vice versa.

With a limited space under the wing in which to integrate the engine, the designer must be conscious of the size of the engine and the maximum allowable engine size may be smaller than that of the optimum engine cycle. The third line in Figure 11 shows the trend line when the fan diameter is constrained to be no greater than that of the baseline $(11.0 \mathrm{ft})$. Meeting the new diameter constraint requires an increase in FPR and a decrease in BPR and OPR, which imposes an additional SFC penalty, although the cumulative noise level at the performance optimum is actually lower. The dark single point indicates the SFC and cumulative noise level of the baseline engine. The location of this point along the Pareto set confirms that the baseline engine has been optimized near the minimum cruise SFC point and has

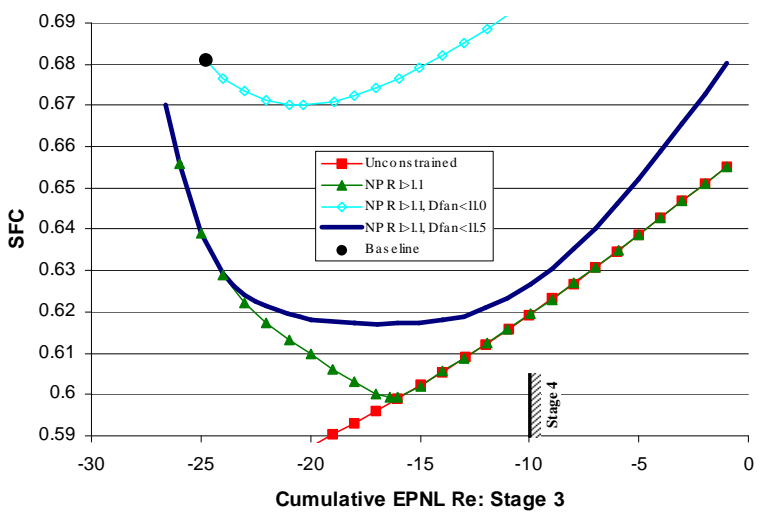

Figure 11. Minimum SFC vs. cumulative noise level. likely been designed for reduced noise levels at the expense of a small increase in SFC.

Finally, the fourth line in Figure 11 shows the trend line with the fan diameter constraint relaxed to 11.5 ft. Allowing a larger fan diameter allows the BPR to be increased and the FPR and OPR to be reduced, and the engine can be optimized at a lower SFC than the baseline. The optimum SFC is quite sensitive to the allowable fan diameter, as a 6 -inch increase in diameter can give an $8 \%$ reduction in SFC. Although the new optimum has a higher cumulative noise level, the larger-diameter design can always be optimized to a lower SFC for a given noise level than the smaller-diameter design, or conversely, a lower cumulative noise level for a given SFC.

\section{B. Swept Stator Benefits}

Swept stator vanes can be employed to reduce the fan noise of an engine, producing a lower total noise level for a given engine design cycle. The effect of swept stators cannot be modelled using the empirical fan noise methods in ANOPP, though, so their benefits would need to be obtained from external sources and incorporated as an adjustment to the predicted fan noise. In a traditional design process, the benefits of swept stators might be assumed to be constant for all engine cycles based on limited test results. In this study, however, the RSEs that were created could model the interactions between the stator sweep angle and the other engine design variables, so the analysis was able to discern differ-

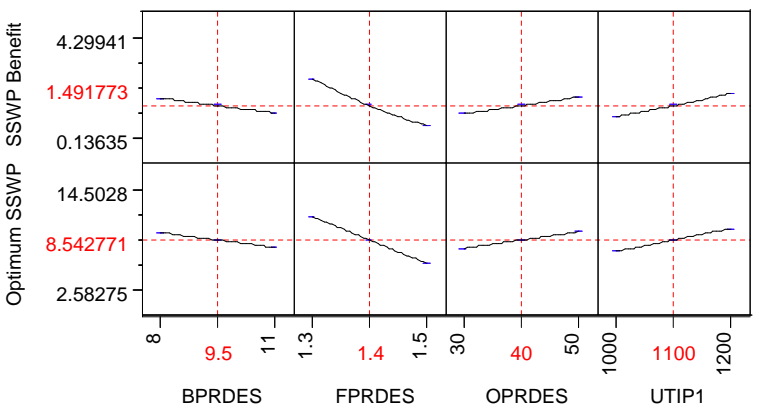

Figure 12. Benefits of stator sweep as a function of engine cycle design. ent swept stator benefits to the sideline, cutback and approach noise, as well as showing how the swept stator benefit varies for different engine cycles.

To show how the benefits of stator sweep vary for different engine cycles, a DOE study was run for the engine cycle design variables which contribute to the cumulative noise levels and the RSEs were used to calculate the noise levels. For each design point, the stator sweep angle was optimized to minimize the cumulative total noise level. Figure 12 shows parametric sensitivity plots for the stator sweep benefit to the total aircraft cumulative noise level, as a function of the engine cycle design variables. Instead of being 
constant for all engines, the noise benefit of stator sweep is actually a function of the engine cycle to which it is applied.

The SFC vs. noise tradeoff study from the previous section was revisited to assess the benefits of swept stators when selecting an optimum engine cycle. Figure 13 shows the difference between the previous trend line with unswept stators (Line 4 from Figure 11), and a new line along which the stator sweep angle was allowed to vary to find the optimum value. This means that the noise is lower for a given SFC, but also for a given target cumulative noise level the engine can be optimized to a lower cruise point SFC.

\section{Robust Engine Design}

It is desirable when designing the engine to allow for a margin of safety in each of the constraints to reduce the risk that the engine will fail to meet the constraints as predicted and force a costly redesign at a later stage in the design process. It also is desirable to allow for a margin of safety in the promised performance and noise levels to reduce the risk that design changes or costly corrections will need to be made to improve the performance or reduce the noise levels to the values that had been previously guaranteed.

\section{Probabilistic RSEs, Baseline Airframe}

A probabilistic DOE study was performed in which the engine design variables were systematically varied, and for each design point a Monte Carlo analysis was performed in which all of the uncertain parameters were varied according to their defined distributions. Each of the uncertain variables was sampled from a normal distribution with a mean equal to the midpoint of the defined variable range, and a standard deviation equal to one quarter of the variable range; with the exception that the turbine cooling mass flow was sampled from a triangular distribution with minimum and most-likely values of 0.25 and a maximum value of 0.30 (Figure 14). Monte Carlo analyses were performed using 10,000 simulations per metric and the resulting outputs from the RSEs were tabulated. This DOE study was similar to those conducted previously, except in this case the responses were not deterministic values for the different metrics, but instead were probability distributions.

For each design point the mean and standard deviation of the distribution were recorded, and used to calculate confidence margins on the metrics. For the cruise point SFC and the noise metrics, the $90 \%$ confidence values were calculated by approximating the empirical probability distributions as normal distributions, while he core NPR and fan diameter were approximated by triangular distributions. Using the results of the new DOE studies, RSEs were created to give the mean, standard deviation and $90 \%$ confidence value as functions of the different engine design variables. Figure 15 shows a sample parametric sensitivity plot for sideline EPNL. By varying the design variables, not only is the mean

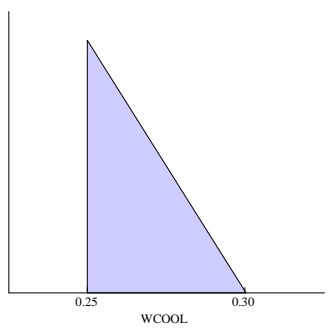

(a) turbine cooling

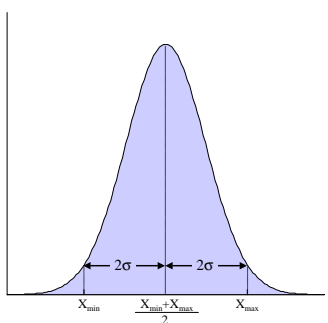

(b) all other uncertain variables

Figure 14. Defined probability distributions for uncertain variables. value of each metric affected as seen previously, but the uncertainty in the values also changes. This means that it is possible to purposely choose values of design variables which reduce the uncertainty in the metrics and increase the confidence that the engine will satisfy the constraints and meet its performance and noise targets. 


\section{Probabilistic Analysis for All Airframes}

From the engine designer's perspective, the airframe design variables could be treated as uncertain variables because the engine designer has little actual control over their values. Representing the airframe variables as distributions of values, rather than deterministic values, allows the engine designer to examine how the engine will perform for a range of different airframe types, or under the influence of uncertainty in the final design choices or aerodynamic performance of a yet-to-be built airframe. To examine these concepts, further analyses were performed in which the airframe variables were treated as uncertain variables and given probability distributions. Uniform distributions were used for these variables to represent the idea that these their values are known for a given aircraft, but would vary

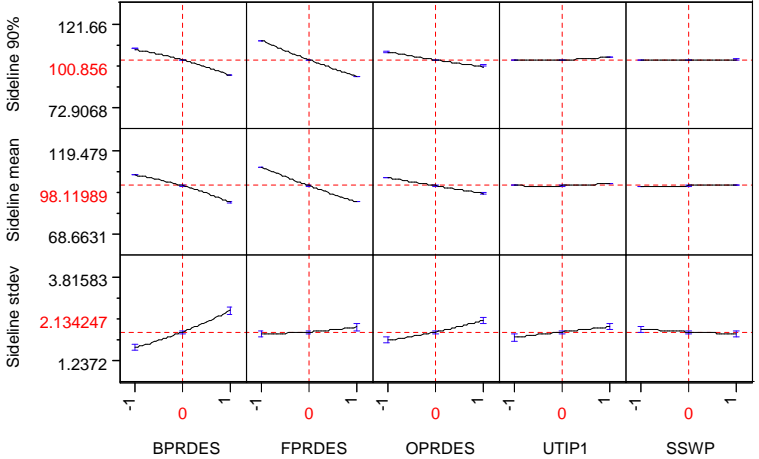

Figure 15. Parametric sensitivity plots for mean, standard deviation and $90 \%$ confidence level of sideline EPNL, baseline airframe. over a range of values when dealing with a number of different airframe designs. A logical check was used to examine the airframe performance metrics and reject the combinations of variables which resulted in invalid airframes, i.e. those that exceeded reasonable values for takeoff and landing field lengths or approach velocity.

\section{Probabilistic RSEs, All Airframes}

A final DOE study was conducted to assess the effect of the engine design variables on the probability distributions of the different metrics; similarly to the DOE study in Section VI.C.1, a Monte Carlo analysis was performed at each design point to arrive at probability distributions for each of the metrics, except in this study the airframe variables were also varied uniformly within their assumed ranges. As discussed in the previous section, combinations of airframe variables which failed to meet reasonable performance metrics were rejected.

\section{SFC Vs. Noise With Confidence}

The new probabilistic RSEs were used to revisit the SFC vs. cumulative noise level trade study of Section VI.A, except that instead of deterministic values for the SFC, noise levels and constraints, the results of the probabilistic analyses were used to find the values with $90 \%$ confidence. In the first case, the means and standard deviations of the metrics for just the baseline engine and airframe were used to define margins of safety for the metrics, i.e. a set of values which must be added to, or subtracted from, the metrics to give $90 \%$ confidence in their predicted values; these margins of safety were assumed constant for all engine cycles to simulate a situation in which computational constraints limit the ability to perform probabilistic analyses for more configurations than just the baseline. In the second case, the new trend line was traced using the RSEs for the $90 \%$ confidence levels of the different metrics for the baseline airframe. The third case used the RSEs for the $90 \%$ confidence levels of the different metrics for all valid airframes in the design space. In each case, the trend line was traced by minimizing the $90 \%$ confidence value of SFC for each $90 \%$ confidence value of cumulative noise, with the requirement that the core NPR and fan diameter constraints be met with $90 \%$ confidence.

The results of the probabilistic SFC vs. cumulative noise trade study are shown in Figure 16. Figure 16(a) plots the $90 \%$ confidence values of SFC and cumulative noise margin, while Figure 16(b) shows the corresponding deterministic values. The first line in each plot shows the previous results of the deterministic trade study (Line 4 from Figure 11), while the second and subsequent lines show the results of the trade study when the $90 \%$ confidence values for the metrics are used instead. The trend line is shifted significantly by the added requirement that the constraints be met with $90 \%$ confidence. To increase the confidence that the constraints will be met, the engine must be designed with a lower BPR and a lower OPR, resulting in an SFC penalty. The cumulative noise level is not adversely affected by the increase in confidence, except that the noise will always be higher for a given target value of SFC. 


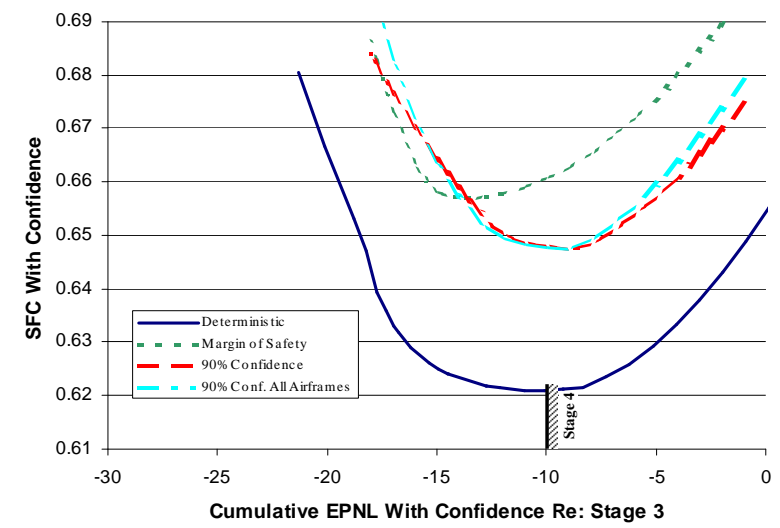

(a) $90 \%$ confidence values

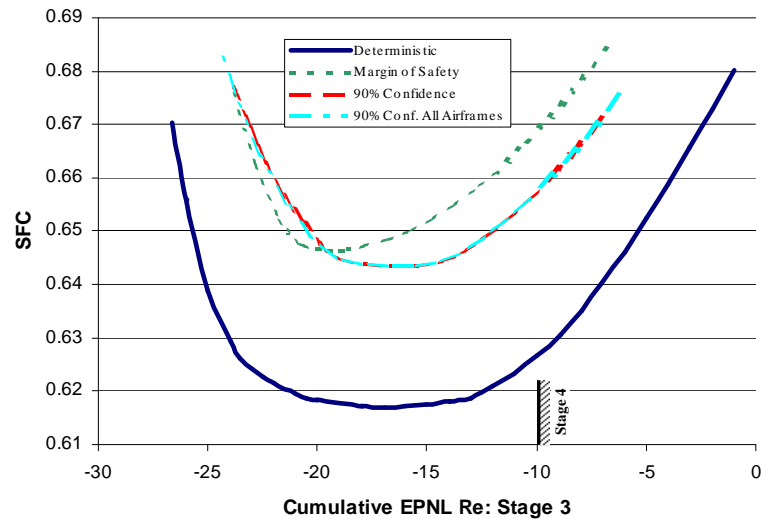

(b) deterministic values

Figure 16. Minimum SFC vs. cumulative noise level.

There is a significant difference in the results when using constant margins of safety, as compared to calculating the variation of standard deviation over the design space. Assuming a constant margin of safety can result in an inaccurate assessment of the confidence levels of the constraints and performance and noise targets. The confidence levels for SFC and the fan diameter are higher than intended, and as a result the engine has been optimized with an SFC value $0.4 \%$ higher than was necessary to achieve the desired $90 \%$ confidence levels for the constraints. Even worse, the confidence levels for the core NPR and cumulative noise are lower than intended, so the optimized design actually has higher risk than intended, with the added danger that the designer may not realize that the risk has been underpredicted.

On the other hand, there is almost no difference between the results for the baseline airframe and the results over all airframes. The $90 \%$ confidence value of the cumulative noise level for all airframes is higher than the corresponding value for just the baseline airframe, but the relationship between the engine design variables and the standard deviation of the cumulative noise follows the same pattern in each case. It is not necessary in this case to make modifications to the design of the engine to improve the robustness with respect to the type of airframe on which it is installed; it would appear that it is sufficient to use the baseline airframe as a platform for the optimization of the engine cycle.

\section{Conclusions}

The analyses of the previous section demonstrated the utility of using metamodels in the conceptual design process as surrogates for computationally intensive analysis methods. Although this study was focused on the prediction of noise levels for conceptual engine and aircraft designs, the same concepts can be applied to a full range of design problems in many disciplines. The advantage of these techniques is clear for high-fidelity analysis methods that take hours or days to run, but the combination of metamodelling combined with risk analysis is useful even when the analysis method is fairly fast, since a robust design optimization process based on finding the optimum design at a given confidence level will inevitably require tens of thousands of runs. For an analysis method that takes just 30 seconds to complete, this would require a week or more to run the optimization.

With the ability to perform extremely rapid evaluations of the design metrics, it is possible to glean much more information out of design trade studies. Instead of running an optimizer and finding a few select points which define the tradeoff between SFC and cumulative noise level, it was possible to plot the trend line for the entire design space, for several different constraint scenarios. It was even possible to plot the complete trend line for probabilistic values of SFC, cumulative noise, fan diameter and core NPR. Being able to visualize results for the entire design space, rather than for a select number of optimized points, can greatly assist the designer in understanding the tradeoffs involved. Since the RSEs represent a global model of the design space, they can also be reused as much as needed without needing to return to the analysis process for additional time-consuming runs.

The greatest difficulty in implementing FPA methods in a conceptual design process proved to be in the generation of a geometric definition of the engine components at sufficient detail to perform the computational 
aerodynamic analyses needed for calculation of the fan tone noise and jet mixing noise levels. During conceptual design, engine cycle performance is usually calculated with one-dimensional analysis methods which require very little knowledge of the actual geometry of the engine components, so additional methods had to be added to the methodology for the purpose of developing the geometric definition at the required level of detail.

The use of an FPA methodology, combined with a method such as RSM which defines clear relationships between the design variables and the output metrics, is a powerful tool for assessing the benefits of technologies which cannot otherwise by accounted for using empirical methods. RSEs were used in this study to not only directly calculate the benefits of fan stator sweep to the cumulative noise level, but to examine how those benefits change for different engine cycles. Furthermore, the ability to rapidly evaluate the technology benefits allows the designer to optimize the engine and technology parameters simultaneously to achieve the most effective application of the new technology.

The analyses in this study demonstrated that the robust design of an aircraft engine is a more complex problem than simply adding a margin of safety to the computed metrics to reduce the risk. This approach was shown to result in a different choice of design point than a more rigorous analysis which accounts for the effects of engine cycle on the variability of the metrics. Choosing the design cycle based on the simpler margin of safety approach can result in a cycle that has been over-designed relative to certain design requirements and under-designed relative to others.

\section{References}

${ }^{1}$ Burleson, C. and Maurice, L., "Aviation and the Environment: Challenges and Opportunities," AIAA/ICAS International Air and Space Symposium and Exposition: The Next 100 Years, American Institute of Aeronautics and Astronautics, 14-17 July 2003

${ }^{2}$ Willshire, Jr, W. L. and Stephens, D. G., "Aircraft Noise Technology for the 21st Century," NOISE-CON 98, Institute of Noise Control Engineering of the USA, 5-8 April 1998, pp. 7-22.

3 "National Research and Development Plan for Aviation Safety, Security, Efficiency, and Environmental Compatibility," National Science and Technology Council Subcommittee on Transportation Research and Development, Nov. 1999.

${ }^{4}$ Thomas, C., Hume, K., and Hooper, P., "Aircraft Noise, Airport Growth and Regional Development," AIAA Paper 2004-2806, American Institute of Aeronautics and Astronautics, May 2004.

${ }^{5}$ Thomas, R. H., Choudhari, M. M., and Joslin, R. D., "Flow and Noise Control: Toward a Closer Linkage," ICAS 2002 Congress, Toronto, CA, 8-13 Sept. 2002.

${ }^{6}$ Hodge, C. G., "Quiet Aircraft Design and Operational Characteristics," Aeroacoustics of Flight Vehicles: Theory and Practice, edited by H. H. Hubbard, Vol. 2, NASA RP-1258, Aug. 1991, pp. 383-414.

7 "Noise Standards: Aircraft Type and Airworthiness Certification," Code of Federal Regulations Title 14, Part 36, Federal Aviation Administration, Department of Transportation, 1 Jan. 1990.

8 "Aircraft Noise," International Civil Aviation Organization (ICAO) Annex 16, Environmental Protection, volume I, Amendment 7 effective 21 March 2002

${ }^{9}$ Olson, E. D. and Mavris, D. N., "An Integrated Low-speed Performance and Noise Prediction Methodology for Subsonic Aircraft," Proceedings of the 6th AIAA/CEAS Aeroacoustics Conference, Lahaina, HI, 12-14 June 2000.

${ }^{10}$ Geiselhart, K. A., "A Technique for Integrating Engine Cycle and Aircraft Configuration Optimization," NASA CR 191602, National Aeronautics and Space Administration, Feb. 1994.

${ }^{11}$ Geiselhart, K. A., Engine Cycle Analysis Program User's Guide, Version 4.0.

${ }^{12}$ Geiselhart, K. A., Caddy, M. J., and Morris, Jr, S. J., "Computer Program for Estimating Performance of Air-Breathing Aircraft Engines," NASA TM 4254, National Aeronautics and Space Administration, May 1991.

${ }^{13}$ Caddy, M. J. and Shapiro, S. R., "NEPCOMP—The Navy Engine Performance Computer Program, Version 1," NADC 74045-30, Naval Air Development Center, April 1975.

${ }^{14}$ Katsanis, T. and McNally, W. D., "Revised FORTRAN Program for Calculating Velocities and Streamlines on the HubShroud Midchannel Stream Surface of an Axial-, Radial-, or Mixed-flow Turbomachine or Annular Duct, I-User's Manual," Nasa tn, National Aeronautics and Space Administration, March 1977.

${ }^{15}$ Vanderplaats, G. N., "CONMIN - A FORTRAN Program for Constrained Function Minimization: User's Manual," NASA TM X-62282, National Aeronautics and Space Administration, Aug. 1973.

${ }^{16}$ Chima, R. V., "Calculation of Multistage Turbomachinery Using Steady Characteristic Boundary Conditions," AIAA Paper 98-0968, American Institute of Aeronautics and Astronautics, Jan. 1998, Also NASA TM-1998-206613.

${ }^{17}$ Chima, R. V., "Calculation of Tip Clearance Effects in a Transonic Compressor Rotor," Journal of Turbomachinery, Vol. 120, No. 1, Jan. 1998, pp. 131-140.

${ }^{18}$ Chima, R. V., "Computational Modeling of Vortex Generators for Turbomachinery," ASME Paper GT-2002-30677, American Society of Mechanical Engineers, April 2002, Also NASA TM-2002-211551.

${ }^{19}$ Chima, R. V., "Comparison of the AUSM+ and H-Cusp Schemes for Turbomachinery Applications," AIAA Paper 20034120, American Institute of Aeronautics and Astronautics, June 2003, Also NASA TM-2003-212457.

${ }^{20}$ Chima, R. V., "Viscous Three-Dimensional Calculations of Transonic Fan Performance," CFD Techniques for Propulsion 
Appliations, AGARD Conference Proceedings No. CP-510, Advisory Group for Aerospace Research and Development, Feb. 1992, pp. 21-1 to 21-19, Also NASA TM-103800.

${ }^{21}$ Chima, R. V., Giel, P. W., and Boyle, R. J., "An Algebraic Turbulence Model for Three-Dimensional Viscous Flows," AIAA Paper 93-0083, American Institute of Aeronautics and Astronautics, Jan. 1993, Also NASA TM-105931.

${ }^{22}$ Chima, R. V. and Yokota, J. W., "Numerical Analysis of Three-Dimensional Viscous Flows in Turbomachinery," AIAA Journal, Vol. 28, No. 5, May 1990, pp. 798-806.

${ }^{23}$ Tweedt, D. L., Chima, R. V., and Turkel, E., "Preconditioning for Numerical Simulation of Low Mach Number ThreeDimensional Viscous Turbomachinery Flows," AIAA Paper 97-1828, American Institute of Aeronautics and Astronautics, June 1997.

${ }^{24}$ Chima, R. V., TCGRID 3-D Grid Generator for Turbomachinery, User's Manual and Documentation, Version 206, NASA Glenn Research Center, Cleveland, OH, Dec. 1999.

${ }^{25}$ Hobbs, D. E. and Weingold, H. D., "Development of Controlled Diffusion Airfoils for Multistage Compressor Applications," Journal of Engineering for Gas Turbines and Power, Vol. 106, No. 4, April 1984, pp. 271-278.

${ }^{26}$ Nerurkar, A. C., Dang, T. Q., Reddy, E. S., and Reddy, D. R., "Design Study of Turbomachine Blades by Optimization and Inverse Techniques," AIAA Paper 96-2555, American Institute of Aeronautics and Astronautics, July 1996.

${ }^{27}$ Damle, S. V., "Throughflow Method for Turbomachines Using Euler Solvers," AIAA Paper 96-0010, American Institute of Aeronautics and Astronautics, Jan. 1996.

${ }^{28}$ McCullers, L. A., FLOPS User's Guide, Version 6.02, NASA Langley Research Center, Hampton, VA, 11 March 2003.

29 "Airworthiness Standards: Transport Category Airplanes," Code of Federal Regulations Title 14, Part 25, Federal Aviation Administration, Department of Transportation, 26 Dec. 2002.

${ }^{30}$ Topol, D. A., "Development and Evaluation of a Coupled Fan Noise Design System," Proceedings of the 3rd AIAA/CEAS Aeroacoustics Conference, American Institute of Aeronautics and Astronautics, 12-14 May 1997.

${ }^{31}$ Topol, D. A. and Eversman, W., "TFaNS Tone Fan Noise Design/Prediction System, Volume II: User's Manual, TFaNS Vers 1.4," NASA CR 1999-208883, National Aeronautics and Space Administration, March 1999.

${ }^{32}$ Roy, I. D. and Eversman, W., "Development of the Inlet and Aft Fan Duct Acoustic Radiation Codes," Informal Report to NASA Lewis under NASA grant NAG3 1678.

${ }^{33}$ Roy, I. D. and Eversman, W., "Improved Finite Element Modeling of the Turbofan Engine Inlet Radiation Problem," Journal of Vibration and Acoustics, Vol. 117, No. 1, Jan. 1995, pp. 109-115.

${ }^{34}$ Bush, R. H., Power, G. D., and Towne, C. E., "WIND: The Production Flow Solver of the NPARC Alliance," Proceedings of the 36th Aerospace Sciences Meeting and Exhibit, American Institute of Aeronautics and Astronautics, 12-15 Jan. 1998.

${ }^{35}$ Khavaran, A., Krejsa, E. A., and Kim, C. M., "Computation of Supersonic Jet Mixing Noise for an Axisymmetric Convergent-Divergent Nozzle," Journal of Aircraft, Vol. 31, No. 3, May-Jun. 1994, pp. 603-609.

${ }^{36}$ Gliebe, P. R., Motsinger, R. E., and Sieckman, A., "High Velocity Jet Noise Source Location and Reduction, Task 6 Supplement-Computer Programs: Engineering Correlation $\left(\mathrm{M}^{*} \mathrm{~S}\right)$, Jet Noise Prediction Method and Unified Aeroacoustic Prediction Model $\left(\mathrm{M}^{*} \mathrm{G}^{*} \mathrm{~B}\right)$ for Nozzles of Arbitrary Shape," FAA RD 76-79, VIa, Federal Aviation Administration, March 1979.

${ }^{37}$ Gillian, R. E., "Aircraft Noise Prediction Program User's Manual," NASA TM 84486, National Aeronautics and Space Administration, Jan. 1983.

${ }^{38}$ Zorumski, W. E., "Aircraft Noise Prediction Program Theoretical Manual," NASA TM 83199, National Aeronautics and Space Administration, Feb. 1982.

${ }^{39}$ Mavris, D. N., DeLaurentis, D. A., Bandte, O., and Hale, M. A., "A Stochastic Approach to Multi-disciplinary Aircraft Analysis and Design," Proceedings of the 36th Aerospace Sciences Meeting and Exhibit, American Institute of Aeronautics and Astronautics, 12-15 Jan. 1998.

${ }^{40}$ Khuri, A. J. and Cornel, J. A., Response Surface Methodology, Virginia Commonwealth University, Boston, 1971.

${ }^{41}$ Box, G. E. P., Hunter, W. G., and Hunter, J. S., Statistics for Experimenters, An Introduction to Design, Analysis, and Model Building, John Wiley \& Sons, New York, 1978.

${ }^{42}$ Auzins, J., "Direct Optimization of Experimental Designs," Proceedings of the 10th AIAA/ISSMO Multidisciplinary Analysis and Optimization Conference, American Institute of Aeronautics and Astronautics, 30 Aug.-1 Sept. 2004.

${ }^{43}$ Neter, J., Kutner, M. H., Nachtsheim, C. J., and Wasserman, W., Applied Linear Statistical Models, Irwin, Chicago, IL.

${ }^{44}$ Heidmann, M. F., "Interim Prediction Method for Fan and Compressor Source Noise," NASA CR X-71763, National Aeronautics and Space Administration, June 1979.

${ }^{45}$ Gliebe, P. R., "The GE90: Quiet by Design: Quieter Aircraft Engines Through Leveraging New Technologies," Presentation for 2003 Berkeley Airport Noise Symposium, Doing The Wright Stuff: 100 years of Aviation and the Environment. 